\title{
Transcription factor organic cation transporter 1 (OCT-1) affects the expression of porcine Klotho $(K L)$ gene
}

\author{
Yan Li ${ }^{1}$, Lei Wang ${ }^{1}$, Jiawei Zhou ${ }^{1}$, Fenge Li ${ }^{\text {corresp. } 1}$ \\ ${ }^{1}$ Key Laboratory of Pig Genetics and Breeding of Ministry of Agriculture \& Key Laboratory of Agricultural Animal Genetics, Breeding and Reproduction of \\ Ministry of Education, Huazhong Agricultural University, Wuhan, PR China \\ Corresponding Author: Fenge Li \\ Email address: lifener@mail.hzau.edu.cn
}

Klotho $(\mathrm{KL})$, originally discovered as an aging suppressor, is a membrane protein that shares sequence similarity with the $\beta$-glucosidase enzymes.Recent reports showed Klotho might play a role in adipocyte maturation and systemic glucose metabolism. However, little is known about the transcription factors involved in regulating the expressionof porcine $K L$ gene. Deletion fragment analysis identified KL-D2 (-418 bp to $-3 \mathrm{bp})$ as the porcine $K L$ core promoter. MARC0022311SNP ( $A$ or $G$ ) in KL intron 1 was detected in Landracex DIV pigs using the Porcine SNP60 BeadChip. The pGL-D2-A and pGL-D2-G were constructed with KLD2 and the intron fragment of different alleles and relative luciferase activity of pGL3-D2-G was significantly higher than that of pGL3-D2-A in the PK cells and ST cells.It was possibly the result of a change in $K L$ binding ability with transcription factor organic cation transporter 1 (OCT-1), which was confirmed using electrophoretic mobility shift assays (EMSA) and chromatin immune-precipitation (ChIP). Moreover, OCT-1 regulated endogenous $K L$ expression by RNA interference experiments. Our study indicates SNP MARC0022311 affects porcine $K L$ expression by regulating its promoter activity via OCT- 1 . 
1 Transcription factor organic cation transporter 1 (OCT-1) affects the 2 expression of porcine Klotho $(K L)$ gene

3 Yan Li ${ }^{1 \#}$, Lei Wang ${ }^{1 \#}$, Jiawei Zhou ${ }^{1}$, Fenge $\mathrm{Li}^{1 *}$

$4 \quad{ }^{1}$ Key Laboratory of Pig Genetics and Breeding of Ministry of Agriculture \& Key Laboratory of

5 Agricultural Animal Genetics, Breeding and Reproduction of Ministry of Education, Huazhong

6 Agricultural University, Wuhan, 430070, PR China

7 Short Title: OCT-1 affects porcine $K L$ expression

$8 \quad$ \#Yan Li and Lei Wang have contributed equally to this work.

9 "corresponding author: Dr. Fenge Li. Affiliation: College of Animal Science, Huazhong

10 Agricultural University, Wuhan, 430070, PR China; Tel: 0086-27-87282091; Fax: 0086-27-

11 87280408; E-mail: lifener@mail.hzau.edu.cn. 


\section{Abstract}

13 Klotho (KL), originally discovered as an aging suppressor, is a membrane protein that shares

14 sequence similarity with the $\beta$-glucosidase enzymes. Recent reports showed Klotho might play a

15 role in adipocyte maturation and systemic glucose metabolism. However, little is known about

16 the transcription factors involved in regulating the expression of porcine $K L$ gene. Deletion

17 fragment analysis identified KL-D2 (-418 bp to $-3 \mathrm{bp})$ as the porcine $K L$ core promoter.

18 MARC0022311 SNP (A or G) in KL intron 1 was detected in Landrace $\times$ DIV pigs using the

19 Porcine SNP60 BeadChip. The pGL-D2-A and pGL-D2-G were constructed with KL-D2 and the

20 intron fragment of different alleles and relative luciferase activity of pGL3-D2-G was

21 significantly higher than that of pGL3-D2-A in the PK cells and ST cells.It was possibly the

22 result of a change in $K L$ binding ability with transcription factor organic cation transporter 1

23 (OCT-1), which was confirmed using electrophoretic mobility shift assays (EMSA) and chromatin immune-precipitation (ChIP). Moreover, OCT-1 regulated endogenous $K L$ expression by RNA interference experiments. Our study indicates SNP MARC0022311 affects porcine $K L$ expression by regulating its promoter activity via OCT-1. 
Introduction

Klotho $(K L)$ gene encodes a membrane protein that shares a sequence similarity with the $\beta$ glucosidase genes and its product may function as part of a signaling pathway that regulates aging and morbidity in age-related diseases (Ko et al., 2013). Mutant mice lacking the $K L$ gene shows multiple aging disorders and a shortened life span (Kuro-o et al., 1997). $K L^{-/-}$mice have the pattern of ectopic calcification certainly contributed by the elevated phosphate and calcium levels (Hu et al., 2011; Ohnishi et al., 2009). KL also acts as a deregulated factor of mineral metabolism in autosomal dominant polycystic kidney disease (Mekahli \& Bacchetta., 2013). Mice that lacked Klotho activity are lean owing to the reduced white adipose tissue accumulation, and are resistant to obesity induced by a high-fat diet (Ohnishi et al., 2011; Razzaque et al., 2012).

$K L$ expression is regulated by thyroid hormone, oxidative stress, long-term hypertension and so on (Koh et al., 2001). Some transcription factors such as peroxisome proliferator-activated receptor gamma (PPAR- $\gamma$ ) also can regulate $K L$ expression (Zhang et al., 2008). A doublepositive feedback loop between PPAR- $\gamma$ and Klotho regulates adipocyte maturation (Chihara et al., 2006; Zhang et al., 2008). Briefly, chromatin immuno-precipitation (ChIP) and gel shift assays find a PPAR-responsive element within the 5'-flanking region of human $K L$ gene. Additionally, PPAR- $\gamma$ agonists increases $K L$ expression in HEK293 cells and several renal epithelial cell lines, while the induction is blocked by PPAR- $\gamma$ antagonists or small interfering RNAs (Zhang et al., 2008). Furthermore, Klotho can induce PPAR- $\gamma$ synthesis during adipocyte maturation (Chihara et al., 2006). However, little is known about the transcription factors 
49

involved in regulating the expression of porcine $K L$ gene.

Several hundreds of thousands of porcine SNPs were discovered using next generation sequencing technologies, and Illumina Inc used these SNPs, as well as others from different public sources, to design a high-density SNP genotyping assay (Ramos et al., 2009). SNP MARC0022311 is one 64,232 SNPs on the Porcine SNP60K BeadChip. In our study, we detected MARC0022311 SNP in some pigs using 60K SNP chip and found that this SNP could affect the transcriptional regulation of KLOTHO gene. To investigate the transcriptional regulation of porcine $K L$ gene, we identified the core promoter of porcine $K L$ gene, analyzed its upstream regulatory elements and revealed that transcription factor OCT1 directly bound to the core promoter region of porcine $K L$ gene and regulated its expression.

\section{Materials and Methods}

\section{Ethics statements}

All animal procedures were performed according to the protocols approved by the Biological Studies Animal Care and Use Committee of Hubei Province, PR China. Sample collection was approved by the ethics committee of Huazhong Agricultural University (No. 30700571 for this study).

\section{MARC0022311 polymorphism in pigs}

Nineteen Landrace $\times$ DIV crossbred pigs were genotyped with the Porcine SNP60 BeadChip (Illumina) using the Infinium HD Assay Ultra protocol, which was conducted under the technical assistance by Compass Biotechnology Corporation. DIV was a synthetic dam line derived by crossing Landrace, Large White, Tongcheng or Meishan pigs. Raw data had high genotyping 
70 quality (call rate $>0.99$ ) and were analyzed with the GenomeStudio software.

\section{In silico sequence analysis}

$K L$ gene sequence ENSSSCG00000009347 was available on the ENSEMBL online website (http://asia.ensembl.org/index.html). We obtained the up-stream sequence of porcine $K L$ gene for promoter prediction. The potential promoter was analyzed using the online neural network promoter prediction (NNPP) (http://www.fruitfly.org/seq tools/promoter.html) and Promoter 2.0 prediction server (http://www.cbs.dtu.dk/services/Promoter/). Transcription factor binding sites were predicted using biological databases (BIOBASE) (http://www.gene-regulation. com/pub/programs.html) with a threshold of 0.90 and TFsearch with a threshold of 85 (Akiyama, 1995). Threshold represents minimum probability of predicted transcription factor.

\section{Cell culture, transient transfection and luciferase assay}

The porcine kidney (PK) cells and swine testis (ST) cells obtained from China Center for Type Culture Collection (CCTCC) were cultured at $37{ }^{\circ} \mathrm{C}$ in a humidified atmosphere of $5 \% \mathrm{CO} 2$ using DMEM supplemented with 10\% FBS (Gibco).

Four $K L$ promoter deletion fragments (KL-D1: -178 bp to $-3 \mathrm{bp}$, KL-D2: -418 bp to -3 bp, KLD3: -599 bp to -3 bp and KL-D4: -835 bp to $-3 \mathrm{bp}$ ) were cloned into $p$ GL3-Basic vector to determine the core promoter region. The plasmids contained pig $K L$ intron 1 fragments (g.1474 A and g.1474 G) followed by KL-D2 promoter (-418 bp to $-3 \mathrm{bp}$ ) were reconstructed, then transfected using lipofectamine 2000 (Invitrogen) into PK cells and ST cells. Plasmid DNA of each well used in the transfection containing $0.8 \mu \mathrm{g}$ of $K L$ promoter constructs and $0.04 \mu \mathrm{g}$ of the internal control vector $p R L-T K$ Renilla/luciferase plasmid. The enzymatic activity of luciferase 
91

92

93

94

95

was then measured with PerkinElmer 2030 Multilabel Reader (PerkinElmer).

\section{RNA interference}

Double-stranded small interfering RNAs (siRNAs) targeting OCT-1 were obtained from GenePharma. Cells were co-transfected with $2 \mu \mathrm{l}$ of siRNA, $0.2 \mu \mathrm{g}$ of reconstructed plasmids using Lipofetamine $2000^{\mathrm{TM}}$ reagent for $24 \mathrm{~h}$. Transfection mixtures were removed, and fresh complete DMEM medium was added to each well. Finally, the enzymatic activity of luciferase was then measured with PerkinElmer 2030 Multilabel Reader (PerkinElmer).

\section{Quantitative real time PCR (qPCR)}

qPCR was performed on the LightCycler ${ }^{\circledR} 480$ (Roche) using SYBR ${ }^{\circledR}$ Green Real-time PCR Master Mix (Toyobo). Primers used in the qPCR were shown in Table 1. qPCR conditions consisted of 1 cycle at $94{ }^{\circ} \mathrm{C}$ for 3 min, followed by 40 cycles at $94{ }^{\circ} \mathrm{C}$ for $40 \mathrm{sec}, 61{ }^{\circ} \mathrm{C}$ for $40 \mathrm{sec}$, and $72{ }^{\circ} \mathrm{C}$ for $20 \mathrm{sec}$, with fluorescence acquisition at $74{ }^{\circ} \mathrm{C}$. All PCRs were performed in triplicate and gene expression levels were quantified relatively to the expression of $\beta$-actin. Analysis of expression level was performed using the $2^{-\Delta \Delta C t}$ method (Livak \& Schmittgen, 2001).

Student's t-test was used for statistical comparisons.

\section{Western blotting}

Western blotting was performed as described previously (Tao et al., 2014). Five $\mu$ g proteins were boiled in $5 \times$ SDS buffer for $5 \mathrm{~min}$, separated by SDS-PAGE, and transferred to PVDF membranes (Millipore). Then, the membranes were blocked with skim milk and probed with anti-KL (ABclonal). $\beta$-actin (Santa Cruz) was used as a loading control. The results were visualized with horseradish peroxidase-conjugated secondary antibodies (Santa Cruz) and 
112 enhanced chemiluminescence.

\section{Electrophoretic mobility shift assays (EMSA)}

114 Nuclear protein of PK and ST cells was extracted with Nucleoprotein Extraction Kit (Beyotime).

115 The oligonucleotides (Sangon) corresponding to the OCT-1 binding sites of $K L$ intron 1 (Table 1)

116 were synthesized and annealed into double strands. The DNA binding activity of OCT-1 protein

117 was detected by LightShift ${ }^{\circledR}$ Chemiluminescent EMSA Kit (Pierce). Ten $\mu$ g nuclear extract was

118 added to 20 fmol biotin-labeled double stranded oligonucleotides, $0.1 \mathrm{mM}$ EDTA, 2.5\% Glycerol,

$1191 \times$ binding buffer, $5 \mathrm{mM} \mathrm{MgCl} 2,50 \mathrm{ng}$ Poly $(\mathrm{dI} \cdot \mathrm{dC})$ and $0.05 \% \mathrm{NP}-40$. In addition, control group added 2 pmol unlabeled competitor oligonucleotides, while the super-shift group added $10 \mu \mathrm{g}$

OCT-1 antibodies (Santa Cruz). The mixtures were then incubated at $24{ }^{\circ} \mathrm{C}$ for $20 \mathrm{~min}$. The

reactions were analyzed by electrophoresis in $5.5 \%$ polyacrylamide gels at $180 \mathrm{~V}$ for $1 \mathrm{~h}$, and then transferred to a nylon membrane. The dried nylon was scanned with GE ImageQuant

LAS4000 mini (GE-Healthcare).

\section{Chromatin immunoprecipitation (ChIP) assay}

ChIP assays were performed using a commercially available ChIP Assay Kit (Beyotime) as previously described (Tao et al., 2015). Briefly, after crosslinking the chromatin with $1 \%$ formaldehyde at $37{ }^{\circ} \mathrm{C}$ for $10 \mathrm{~min}$ and neutralizing with glycine for $5 \mathrm{~min}$ at room temperature, PK and ST cells were washed with cold PBS, scraped and collected on ice. Then, cells were harvested, lysed and treated by sonication. Nuclear lysates were processed 20 times for $10 \mathrm{sec}$ with 20 min intervals on ice water using a Scientz-IID (Scientz). An equal amount of chromatin was immune-precipitated at $4{ }^{\circ} \mathrm{C}$ overnight with at least $1.5 \mu \mathrm{g}$ of OCT-1 antibody (Santa Cruz) 
133 and normal mouse IgG antibody (Millipore). Immune-precipitated products were collected after

134 incubation with Protein $\mathrm{A}+\mathrm{G}$ coated magnetic beads. The beads were washed, and the bound

135 chromatin was eluted in ChIP elution buffer. Then the proteins were digested with Proteinase K

136 for $4 \mathrm{~h}$ at $45{ }^{\circ} \mathrm{C}$. The DNA was purified using the AxyPrep PCR Cleanup Kit (Axygen). The

137 DNA fragment of OCT-1 binding sites in $K L$ intron 1 was amplified with the specific primers

138 (Table 1). The PCR procedure was executed with 36 rounds and in the linear range, ChIP assay

139 had 3 biological replicates.

140 Statistical analysis

141 Statistical analyses based on two-tailed Student's t-tests were performed using the Statistical

142 Package for the Social Sciences software. Significance was determined at a 95\% confidence

143 interval. All data were expressed as the mean \pm standard deviation (S.D.).

144 Results

145 MARC0022311 status in pigs

146 MARC0022311 in KL intron 1 appeared a polymorphism (A or G) in 19 Landrace $\times$ DIV pigs, 147 with 12 AA pigs and AG pigs genotyped using the Illumina PorcineSNP60 chip (Supplementary 148 dataset). The SNP (MARC0022311) in pig $K L$ intron 1 was renamed as $K L$ g.1474 $\mathrm{A}>\mathrm{G}$ 149 according to the standard mutation nomenclature (den Dunnen \& Antonarakis, 2000).

\section{Identification of promoter region of the porcine $K L$ gene}

151 An 833 bp contig in 5' flanking region of pig $K L$ gene was obtained by PCR. To determine the

promoter region, four promoter deletions (KL-D1, KL-D2, KL-D3 and KL-D4) were cloned into 
Luciferase activity analysis in both PK and ST cells revealed that KL-D2 (-418 bp to -3 bp) was essential for its transcriptional activity and was defined as the $K L$ promoter region (Fig. 1B).

\section{MARC0022311 SNP affects the $K L$ expression}

Intron SNPs could not change the amino acid sequence, but might alter gene promoter activity by affecting the binding ability of transcription factors (Van Laere et al., 2003). The plasmids contained the wild-type A (g.1474 A) or mutant G (g.1474 G) sequence followed by KL-D2 were named as pGL3-D2-A and pGL3-D2-G, respectively. Then recombinant DNA fragments were inserted in the downstream of the luc+ gene between the KpnI and HindIII sites. Results showed that luciferase activity of $p G L 3-D 2-G$ was significantly higher than $p G L 3-D 2-A$ in both PK cells $(P<0.05)$ and ST cells $(P<0.01)$ (Fig. 2A), and indicated that MARC0022311 SNP changed the binding ability of certain regulatory elements affected KL promoter activity.

The SNP (MARC0022311) located in the first intron of $K L$ gene $(+1474 \mathrm{bp})$ was predicted to change the binding ability of OCT-1 by BIOBASE and TFsearch (Fig. S1). After silencing OCT1 using siRNAs in PK and ST cells, luciferase activity of $p G L 3-D 2-G$ was significantly lower than $p G L 3-D 2-A(P<0.05)$ (Figs. 2B and 2C). Furthermore, compared with the negative control, the luciferase activity of $p G L 3-D 2-A$ was significantly decreased $(P<0.05)$ (Figs. 2B and 2C). Thus, MARC0022311 regulated the promoter activity via OCT-1.

However, inhibition of $O C T-1$ expression significantly suppressed $K L$ expression in PK and ST cells $(P<0.05)$ (Fig. 3), possibly because OCT-1 could stimulate $K L$ expression by binding $K L$ gene at other sites.

\section{Transcription factor OCT-1 binds to the $K L$ intron 1 both in vitro and in vivo}


175 To address whether KL intron 1 contained OCT-1 binding sites in vitro, we used two

176 oligonucleotides (A allele and G allele oligonucleotides) as porcine OCT-1 probes in EMSA.

177 EMSA revealed a highly specific interaction with allele A oligonucleotide, and a 100 fold excess

178 of mutant allele G oligonucleotide could not outcompete the interaction (Fig. 4A). A super-shift

179 was obtained when nuclear extracts from PK and ST cells were incubated with OCT-1 antibodies,

180 providing further biochemical evidence for the presence of OCT-1 in vitro (Fig. 4A). We found

181 the $K L$ genotype at g.1474 A>G locus was AA in PK and ST cells by PCR-sequencing,

182 indicating the endogenous binding of OCT-1 to $K L$ in above two cell lines (Fig. S2). The

183 chromatin was immune-precipitated using an OCT-1 antibody and DNA fragments of the

184 expected size were used as a template to perform PCR amplification. ChIP analysis showed that

185 OCT-1 interacted with $K L$ intron 1 (Fig. 4B). These results showed that transcription factor

186 OCT-1 bound to $K L$ intron 1 both in vitro and in vivo.

187

\section{Discussion}

$K L$ gene encodes a type-I membrane protein that is related to beta-glucosidases (Ko et al., 2013).

$K L$ may function as part of a signaling pathway that regulates morbidity in age-related diseases such as atherosclerosis and cardiovascular disease, and mineral metabolism diseases such as ectopic calcification (Ko et al., 2013; Kuro-o et al., 1997; Hu et al., 2011; Ohnishi et al., 2009).

Overexpression of $K L$ in the preadipocyte 3T3-L1 cell line can induce expression of several adipogenic markers, including $P P A R \gamma, \mathrm{CCAAT} / \mathrm{enhancer}$ binding protein alpha $(C / E B P \alpha)$ and CCAAT/enhancer binding protein delta $(C / E B P \delta)$, and facilitate the differentiation of preadipocytes into mature adipocytes (Chihara et al., 2006). Eliminating $K L$ function from mice 
196

197

198

199

200

201

202

203

204

205

206

207

208

209

210

211

212

213

214

215

216

results in the generation of lean mice with almost no detectable fat tissue, and induces a resistance to high-fat-diet-stimulated obesity (Razzaque et al., 2012; Ohnishi et al., 2011).

Here we found the SNP MARC0022311 located in KL intron 1 in the tested pigs (Supplementary dataset). A number of SNPs are proved to have major effects on the phenotypic variations (Markljung et al., 2009; Milan et al., 2000; Ren et al., 2011; Van Laere et al., 2003). Previous reports shows that a $\mathrm{G}$ to A transition in intron 3 of porcine insulin-like growth factor 2 (IGF2) affects the binding of ZBED6 and significantly up-regulated $I G F 2$ expression in skeletal muscle (Markljung et al., 2009; Van Laere et al., 2003). We predicted the SNP MARC0022311 located in $K L$ intron 1 could change the binding ability of transcription factors including OCT1 by BIOBASE and TFsearch online software (Fig. S1).

The Octamer-binding proteins (OCTs) are a group of highly conserved transcription factors that specifically bind to the octamer motif (ATGCAAAT) and closely related sequences that are found in promoters and enhancers (Zhao, 2013). OCT1 regulates the expression of a variety of genes, including immunoglobulin genes (Dreyfus, Doyen \& Rougeon, 1987), $\beta$-casein gene (Zhao, Adachi \& Oka, 2002), miR-451/ AMPK signaling (Ansari et al., 2015), sex-determining region Y gene (Margarit et al., 1998), synbindin - related ERK signaling (Qian et al., 2015). In the present study, the pGL3-basic was used as the negative control and inserted core promoter fragment with wild-type and mutant-type intron fragments (pGL3-D2-A, pGL3-D2-G). We wanted to check whether there was different in fluorescent activity between two kinds of plasmids ((Fig 2A)). The pGL3-D2 was used as control and it did not contain intron fragments, and we wanted to verify whether the transcription factor binding to the inserted intron fragment 
217 was the activator or inhibitor (Fig 2B-2C). The luciferase activity of pGL3-D2-G was 218 significantly higher than pGL3-D2-A (Fig 2A) and the following OCT-1 RNAi results showed 219 that luciferase activity of pGL3-D2-G significantly decreased in the scrambled and the pGL3220 D2-A in PK cells and ST cells (Fig 2B-C). The G allele missed one binding sites compared to the

221 A allele ( $\mathrm{G}$ allele had 2 binding sites, while A allele had 3 binding sites) (Fig S1), and displayed 222 a higher luciferase activity than A allele (Fig 2A), suggesting that at this site OCT1 was a 223 repressor. Therefore, we supposed that OCT-1 could bind to the first intron of $K L$ when the SNP was allele $\mathrm{A}$, and then depressed activity of $K L$ promoter.

However, the expression of KL was significantly inhibited after silencing OCT-1. There were several OCT-1 binding sites in porcine KL intron 1 (36324 bp in length) predicted by BIOBASE and TFsearch online software (Fig. S3A). ChIP analysis showed that OCT-1 interacted with all of three tested regions (1395 bp to $1525 \mathrm{bp}, 14322 \mathrm{bp}$ to $14436 \mathrm{bp}, 30970 \mathrm{bp}$ to $31141 \mathrm{bp}$ ) in PK 229 cells (Fig. S3B). It was possible that there was a synergetic effect between the binding sites. Our 230 aim was to detect the difference of OCT-1 binding sites within two alleles which might change KL gene expression. It was certain that A allele created a novel OCT-1 binding site within the flanking region of MARC0022311 SNP by online prediction (Fig S1), which was further confirmed by dual-luciferase reporter assay system (Fig 2) and EMSA (Fig 4A) . In consequence, we hypothesized that OCT1 could dimerise with the chromosome leading to stable binding of the DNA (Tommy et al., 2011; Zabet \& Adryan, 2015). In our study, OCT1 could act as an activator and the presence of the third site in the A allele could disrupt the binding of the dimmer leading to lower activity of the A allele. 
238 Klotho physiologically regulates mineral and energy metabolism by influencing the activities of 239 fibroblast growth factors (FGFs) including FGF-2, FGF-19, FGF-23 and their receptors (FGFRs)

240 (Guan et al., 2014; Razzaque et al., 2009; Wu et al., 2008). Taken together, KL exerts its 241 function via OCT-1 - KL- FGF- FGFR pathway.

\section{Conclusions}

243 In summary, SNP MARC0022311 affected OCT-1 binding ability with the KL promoter. And 244 the $K L$ promoter activity was significantly decreased in allele A of MARC0022311 compared with allele G. Our study indicated SNP MARC0022311 affected porcine $K L$ expression by 246 regulating its promoter activity via OCT-1.

\section{Acknowledgements}

248 We are grateful to Compass Biotechnology Corporation for technical assistance with Illumina 249 SNP analysis. The authors also acknowledge the farmers for providing pig samples.

\section{References}

252

253

Akiyama Y. 1995. TFSEARCH: searching transcription factor binding sites. Real World Computing Partnership, Japan

Ansari KI, Ogawa D, Rooj AK, Lawler SE, Krichevsky AM, Johnson MD, Chiocca EA, Bronisz A, Godlewski J. 2015. Glucose-based regulation of miR-451/AMPK signaling depends on the OCT1 transcription factor. Cell Reports 11(6):902-909

DOI 
258 Chihara Y, Rakugi H, Ishikawa K, Ikushima M, Maekawa Y, Ohta J, Kida I, Ogihara T. 2006.

259 Klotho protein promotes adipocyte differentiation. Endocrinology 147(8):3835-3842 DOI 10.1210/en.2005-1529.

Den Dunnen JT, Antonarakis SE. 2000. Mutation nomenclature extensions and suggestions to describe complex mutations: a discussion. Human Mutation 15:7-12 DOI 10.1002/ (SICI)1098 -1004 (200001)15:1<7::AID-HUMU4>3.0.CO;2-N.

Dreyfus M, Doyen N, Rougeon F. 1987. The conserved decanucleotide from the immunoglobulin heavy chain promoter induces a very high transcriptional activity in Bcells when introduced into a heterologous promoter. EMBO Journal 6:1685-1690.

Guan X, Nie L, He T, Yang K, Xiao T, Wang S, Huang Y, Zhang J, Wang J, Sharma K, Liu Y, Zhao J. 2014. Klotho suppresses renal tubulo- interstitial fibrosis by controlling basic fibroblast growth factor-2 signalling. The Journal of Pathology 234(4):560-572 DOI 10.1002/path.4420.

Hu MC, Shi M, Zhang J, Quiñones H, Griffith C, Kuro-o M, Moe OW. 2011. Klotho deficiency causes vascular calcification in chronic kidney disease. Journal of the American Society of Nephrology 22(1):124-136 DOI 10.1681/ASN.2009. 2013.The association of Klotho gene polymorphism with the mortality of patients on maintenance dialysis. Clinical Nephrology 80(4):263-269 DOI 10.5414/CN107800.

Koh N, Fujimori T, Nishiguchi S, Tamori A, Shiomi S, Nakatani T, Sugimura K, Kishimoto 278 T, Kinoshita S, Kuroki T, Nabeshima Y. 2001. Severely reduced production of Klotho in 
human chronic renal failure kidney. Biochemical and Biophysical Research Communications 280:1015-1020 DOI 10.1006/bbrc.2000.4226. ageing. Nature 390(6655):45-51 DOI 10.1038/36285.

Livak KJ, Schmittgen TD. 2001. Analysis of relative gene expression data using real-time

Markljung E, Jiang L, Jaffe JD, Mikkelsen TS, Wallerman O, Larhammar M, Zhang X, Wang

G, Hjälm G, Andersson L. 2009. ZBED6, a novel transcription factor derived from a domesticated DNA transposon regulates IGF2 expression and muscle growth. PLOS Biology 7(12): e1000256 DOI 10.1371/journal.pbio.1000256.

Margarit E, Guillén A, Rebordosa C, Vidal-Taboada J, Sánchez M, Ballesta F, Oliva R. 1998. Identification of conserved potentially regulatory sequences of the SRY gene from 10 
298

299

300

301

302

303

304

305

306

307

308

309

310

311

312

313

314

315

316

317

318

Mekahli D, Bacchetta J. 2013.From bone abnormalities to mineral metabolism dysregulation in autosomal dominant polycystic kidney disease. Pediatric Nephrology 28:2089-2096 DOI $10.1007 / \mathrm{s} 00467-012-2384-5$.

Milan D, Jeon JT, Looft C, Amarger V, Robic A, Thelander M, Rogel-Gaillard C, Paul S, Iannuccelli N, Rask L, Ronne H, Lundström K, Reinsch N, Gellin J,Kalm E, Roy PL, Chardon P, Andersson L. 2000. A mutation in PRKAG3 associated with excess glycogen content in pig skeletal muscle.Science 288(5469):1248-51.

Ohnishi M, Kato S, Akiyoshi J, Atfi A, Razzaque MS. 2011. Dietary and genetic evidence for enhancing glucose metabolism and reducing obesity by inhibiting klotho functions. The FASEB Journal 25(6): 2031-2039 DOI 10.1096/fj.10-167056.

Ohnishi M, Nakatani T, Lanske B, Razzaque MS. 2009. Reversal of mineral ion homeostasis and soft-tissue calcification of klotho knockout mice by deletion of vitamin D 1alphahydroxylase. Kidney International 75:1166-1172 DOI 10.1038/ki.2009.24.

Qian J, Kong X, Deng N, Tan P, Chen H, Wang J, Li Z, Hu Y, Zou W, Xu J, Fang JY. 2015. OCT1 is a determinant of synbindin-related ERK signalling with independent prognostic significance in gastric cancer. Gut 64(1):37-48 DOI 10.1136/gutjnl-2013-306584.

Ramos AM, Crooijmans RP, Affara NA, Amaral AJ, Archibald AL, Beever JE, Bendixen C, Churcher C, Clark R, Dehais P, Hansen MS, Hedegaard J, Hu ZL, Kerstens HH, Law AS, Megens HJ, Milan D, Nonneman DJ, Rohrer GA, Rothschild MF, Smith TP,Schnabel RD, Van Tassell CP, Taylor JF, Wiedmann RT, Schook LB, Groenen MA. 2009. Design of 
a high density SNP genotyping assay in the pig using SNPs identified and characterized by next generation sequencing technology. PLoS One 4(8):e6524 DOI: 10.1371/journal.pone.0006524.

Razzaque MS. 2009. The FGF23-Klotho axis: endocrine regulation of phosphate homeostasis. Nature Reviews Endocrinology 5(11): 611-619 DOI 10.1038/nrendo.2009.196.

Razzaque MS. 2012. The role of Klotho in energy metabolism. Nature Reviews Endocrinology 8(10):579-587 DOI 10.1038/nrendo.2012.75.

Ren J, Duan Y, Qiao R, Yao F, Zhang Z, Yang B, Guo Y, Xiao S, Wei R, Ouyang Z, Ding N, Ai H, Huang L. 2011.A missense mutation in PPARD causes a major QTL effect on ear size in pigs. PLoS Genetics 7(5):e1002043 DOI 10.1371/journal.pgen.1002043.

Tao H, Mei S, Zhang X, Peng X, Yang J, Zhu L, Zhou J, Wu H, Wang L, Hua L, Li F. 2014. Transcription factor $\mathrm{C} / \mathrm{EBP} 3$ and 173 -Estradiol promote transcription of the porcine p53 gene. The International Journal of Biochemistry \& Cell Biology 47:76-82

DOI 10.1016/j.biocel.2013.12.002.

Tao H, Wang L, Zhou J, Pang P, Cai S, Li J, Mei S, Li F. 2015. The transcription factor ccaat/enhancer binding protein $\beta(\mathrm{C} / \mathrm{EBP} \beta)$ and miR-27a regulate the expression of porcine Dickkopf2 (DKK2). Scientific Reports 5:17972 DOI 10.1038/srep17972.

Tommy K, Xiao Y L, Peter J. S, Sean T, John A. S, Mark D. B, Michael B. E. 2011. Quantitative Models of the Mechanisms That Control Genome-Wide Patterns of Transcription Factor Binding during Early Drosophila Development. PLoS Genet 7(2): e1001290. DOI: $10.1371 /$ journal.pgen.1001290. 
340 Van Laere AS, Nguyen M, Braunschweig M, Nezer C, Collette C, Moreau L, Archibald

341 AL, Haley CS, Buys N, Tally M, Andersson G, Georges M, Andersson L. 2003. A

342 regulatory mutation in IGF2 causes a major QTL effect on muscle growth in the pig.

$343 \quad$ Nature 25 (6960):832-836 DOI 10.1038/nature02064.

344 Wu X, Lemon B, Li X, Gupte J, Weiszmann J, Stevens J, Hawkins N, Shen W, Lindberg R, Chen

345 JL, Tian H, Li Y. 2008. C-terminal tail of FGF19 determines its specificity toward Klotho

346 co-receptors. The Journal of Biological Chemistry 283(48):33304-33309 DOI

$347 \quad$ 10.1074/jbc.M803319200.

348 Zabet NR, Adryan B. 2015. Estimating binding properties of transcription factors from genome-

349 wide binding profiles. Nucleic Acids Research 43 (1): 84-94 DOI: 10.1093/nar/gku1269.

350 Zhang H, Li Y, Fan Y, Wu J, Zhao B, Guan Y, Chien S, Wang N. 2008.Klotho is a target gene of

$351 \quad$ PPAR-gamma. Kidney International 74(6):732-739 DOI 10.1038/ki.2008.244.

352 Zhao FQ. 2013. Octamer-binding transcription factors: genomics and functions. Frontiers in 353 Bioscience (Landmark Ed), 18: 1051-1071.

354 Zhao FQ, Adachi K, Oka T. 2002. Involvement of Oct-1 in transcriptional regulation of beta355 casein gene expression in mouse mammary gland. Biochimica et Biophysica Acta 1577: 27-

$356 \quad 37$ DOI 10.1016/S0167-4781(02)00402-5. 
358

359

360

361

362

363

364

365

366

367

368

369

370

371

372

373

374

375

376

377

378

\section{Figure Legends}

Fig. 1: Deletion analysis of pig $K L$ promoter. (A) Schematic diagram of $K L$ promoter , MARC0022311 (KL g.1474 A>G) and OCT-1 binding site in intron 1. (B) Promoter activities of a series of deleted constructs determined by luciferase assay. Left panel, relative location of four deletion fragments. The nucleotides were numbered from the potential transcriptional start site assigned as +1 . Right panel, the relative luciferase activities of four reconstructed vector contained sequence from KL-D1 to KL-D4. ${ }^{* * *} P<0.001$. The luciferase activity was relative to the Renilla luciferase.

Fig. 2: MARC0022311 in pig $K L$ intron 1 affected promoter activity in PK and ST cells. (A) Luciferase assays of reporter constructs using pig KL-D2 promoter and intron 1 fragments (g.1474 A and g.1474 G). (B) Luciferase detection after co-transfection of OCT-1 siRNA with $p G L 3-D 2-A$ and $p G L 3-D 2-G$ in PK cells. (C) Luciferase detection after co-transfection of OCT-1 siRNA with $p G L 3-D 2-A$ and $p G L 3-D 2-G$ in ST cells. ${ }^{*} P<0.05$. $* * P<0.01$. The pGL3-basic was used as the negative control.

Fig. 3: OCT-1 up-regulated $K L$ expression by RNAi. (A) PK cells were treated with $2 \mu$ OCT-1 siRNA and $2 \mu \mathrm{l}$ NC for $24 \mathrm{~h}$. Knockdown of $O C T-1$ was confirmed by qPCR. $K L$ mRNA and protein expressions were analyzed by qPCR and Western blotting. (B) ST cells were treated with $2 \mu \mathrm{l} O C T-1$ siRNA and $2 \mu \mathrm{l} \mathrm{NC}$ for $24 \mathrm{~h}$. Knockdown of OCT-1 was confirmed by qPCR analysis. $K L$ mRNA and protein expressions were analyzed by qPCR and Western blotting. * $P<0.05$. ** $P<0.01$. Relative mRNA expression was relative to the expression of $\beta$-actin.

Fig. 4: Binding of OCT-1 with $K L$ intron 1 was analyzed by EMSA and ChIP. (A) The probe 
379 was incubated with nuclear extract in the absence or presence of 100 -fold excess of various 380 competitor probes (mutant or non-labeled probe) or anti-OCT-1. The specific super-shift (DNA381 protein-antibody complex) bands were both observed in PK and ST cells. The sequences of 382 various probes were demonstrated under the panel. (B) ChIP assay of OCT-1 binding to the $K L$ intron 1 in PK cells and ST cells. The interaction of OCT-1 in vivo with $K L$ intron region was determined by chromatin immunoprecipitation analysis. DNA isolated from immune-precipitated material was amplified by PCR to amplify $K L$ fragement. Total chromatin was used as the input. Normal mouse IgG was used as a negative control. 
388 Table 1. Primers and DNA oligos used in this study. 


\section{Supplementary files}

390

391

392

393

Fig. S1: Transcription factor binding site prediction of the procine $K L$ intron 1 containing MARC0022311 (KL g.1474 A>G). Quadrilateral frame indicated the substitutions and extra binding site of OCT-1. (A) Predicted by BIOBASE online software with Match - 1.0 Public which is a weight matrix-based program for predicting transcription factor binding sites (TFBS) in DNA sequences. (B) Predicted by TFserach online software of vertebrate database.

Fig. S2: Genotyping results of MARC0022311. (A) PK cells. (B) ST cells. The X axis represented the position of bases and the $\mathrm{Y}$ axis represented fluorescent activity. The greater probability of corresponding base had the higher the fluorescence activity. MARC0022311 was marked in gray backgound.

Fig. S3. OCT-1 binding sites in the porcine $K L$ intron 1. (A) Frequency distribution of the predicted OCT-1 binding sites. X-axis indicated the length of the porcine $K L$ intron 1 in bp. $\mathrm{Y}$ axis was the frequency of the predicted OCT-1 binding sites. (B) ChIP analysis of three candidate OCT-1 binding sites (1395 bp to 1525 bp, 14322 bp to 14436 bp, 30970 bp to 31141 bp) in $K L$ intron 1 in PK cells. Primers used for ChIP-PCR was shown in Table 1. Input and R were positive control, while IgG was the negative control.

Supplementary dataset: SNP genotyping results in 19 Landrace $\times$ DIV pigs using the Porcine SNP60 BeadChip (Illumina). DIV was a synthetic line derived by crossing Landrace, Large White, Tongcheng or Meishan pigs. 


\section{1}

Deletion analysis of pig $K L$ promoter.

(A) Schematic diagram of KL promoter , MARC0022311 ( $K \mathrm{~L}$ g.1474 A>G) and OCT-1 binding site in intron 1. (B) Promoter activities of a series of deleted constructs determined by luciferase assay. Left panel, relative location of four deletion fragments. The nucleotides were numbered from the potential transcriptional start site assigned as +1 . Right panel, the relative luciferase activities of four reconstructed vector contained sequence from KL-D1 to KL-D4. *** $P<0.001$.

A

B
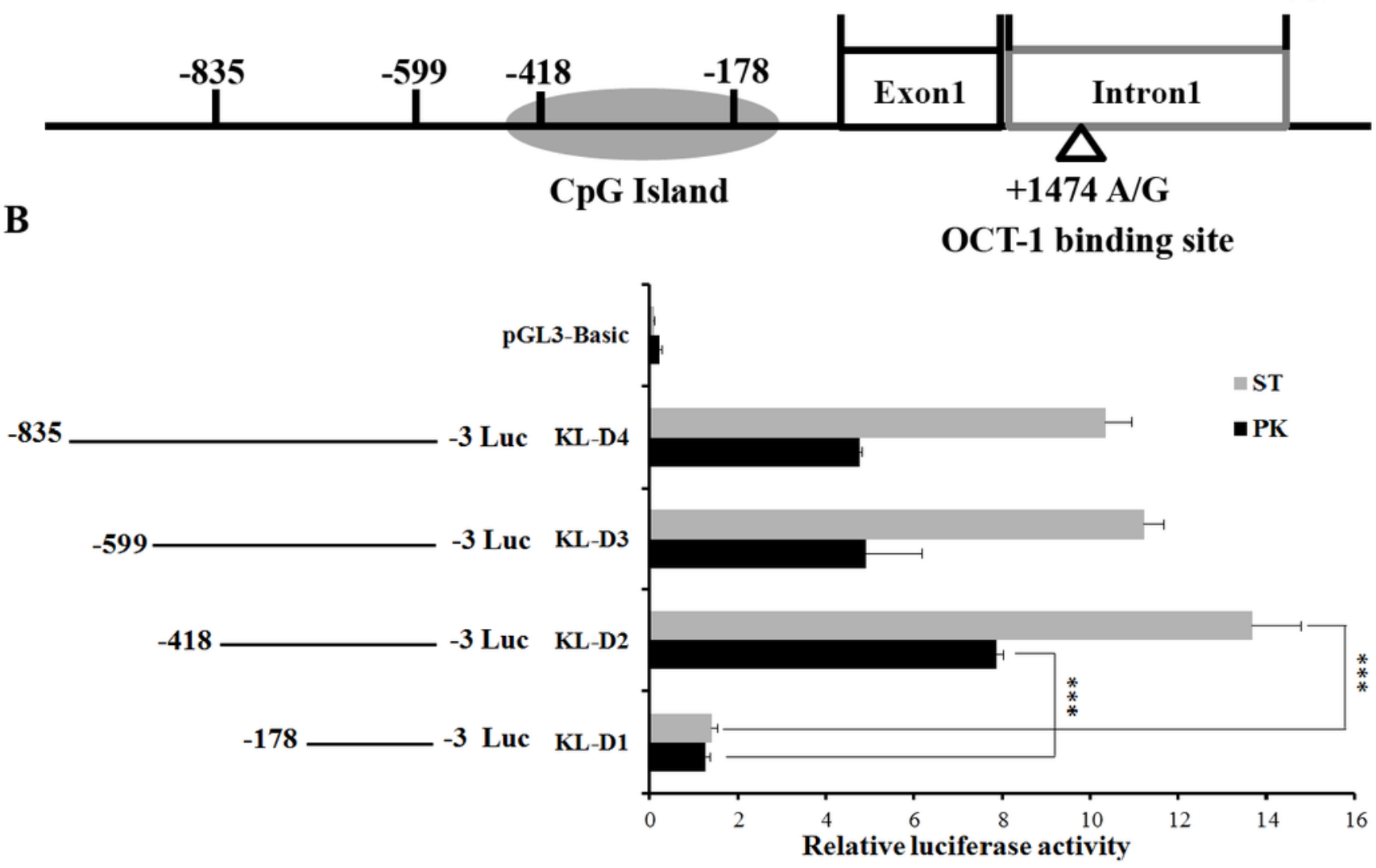
2

MARC0022311 in pig KL intron 1 affected promoter activity in PK and ST cells.

(A) Luciferase assays of reporter constructs using pig KL-D2 promoter and intron 1 fragments (g.1474 A and g.1474 G). (B) Luciferase detection after co-transfection of OCT-1 siRNA with pGL3-D2-A and pGL3-D2-G in PK cells. (C) Luciferase detection after co-transfection of OCT-1 siRNA with $p G L 3-D 2-A$ and $p G L 3-D 2-G$ in ST cells. $* P<0.05$. ** $P<0.01$.

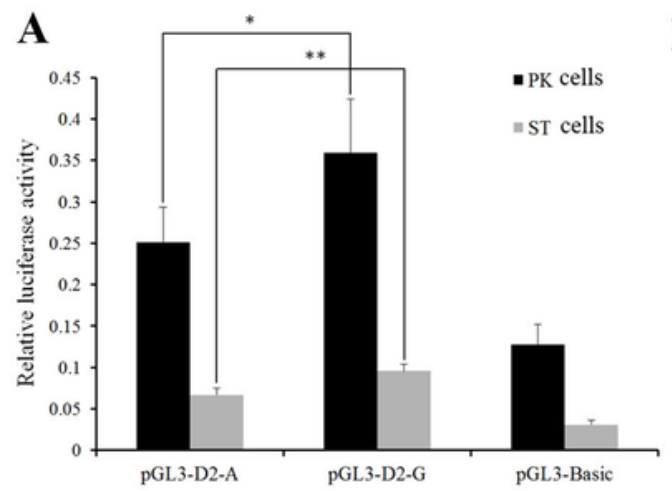

B

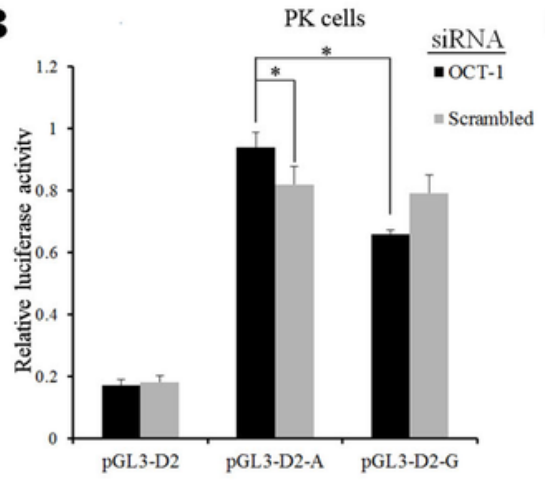

C

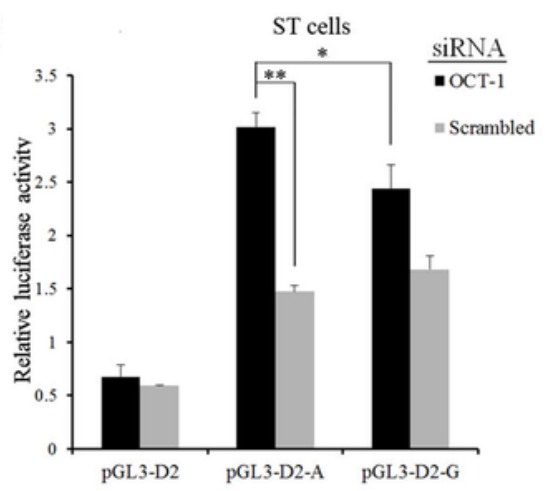


3

OCT-1 up-regulated $K L$ expression by RNAi.

(A) PK cells were treated with $2 \mu \mathrm{l}$ OCT-1 siRNA and $2 \mu \mathrm{l} \mathrm{NC}$ for $24 \mathrm{~h}$. Knockdown of OCT-1 was confirmed by qPCR. KL mRNA and protein expressions were analyzed by qPCR and Western blotting. (B) ST cells were treated with $2 \mu \mathrm{IOCT}-1$ siRNA and $2 \mu \mathrm{NC}$ for $24 \mathrm{~h}$. Knockdown of OCT-1 was confirmed by qPCR analysis. KL mRNA and protein expressions were analyzed by $\mathrm{qPCR}$ and Western blotting. $* P<0.05$. ${ }^{*} P<0.01$.
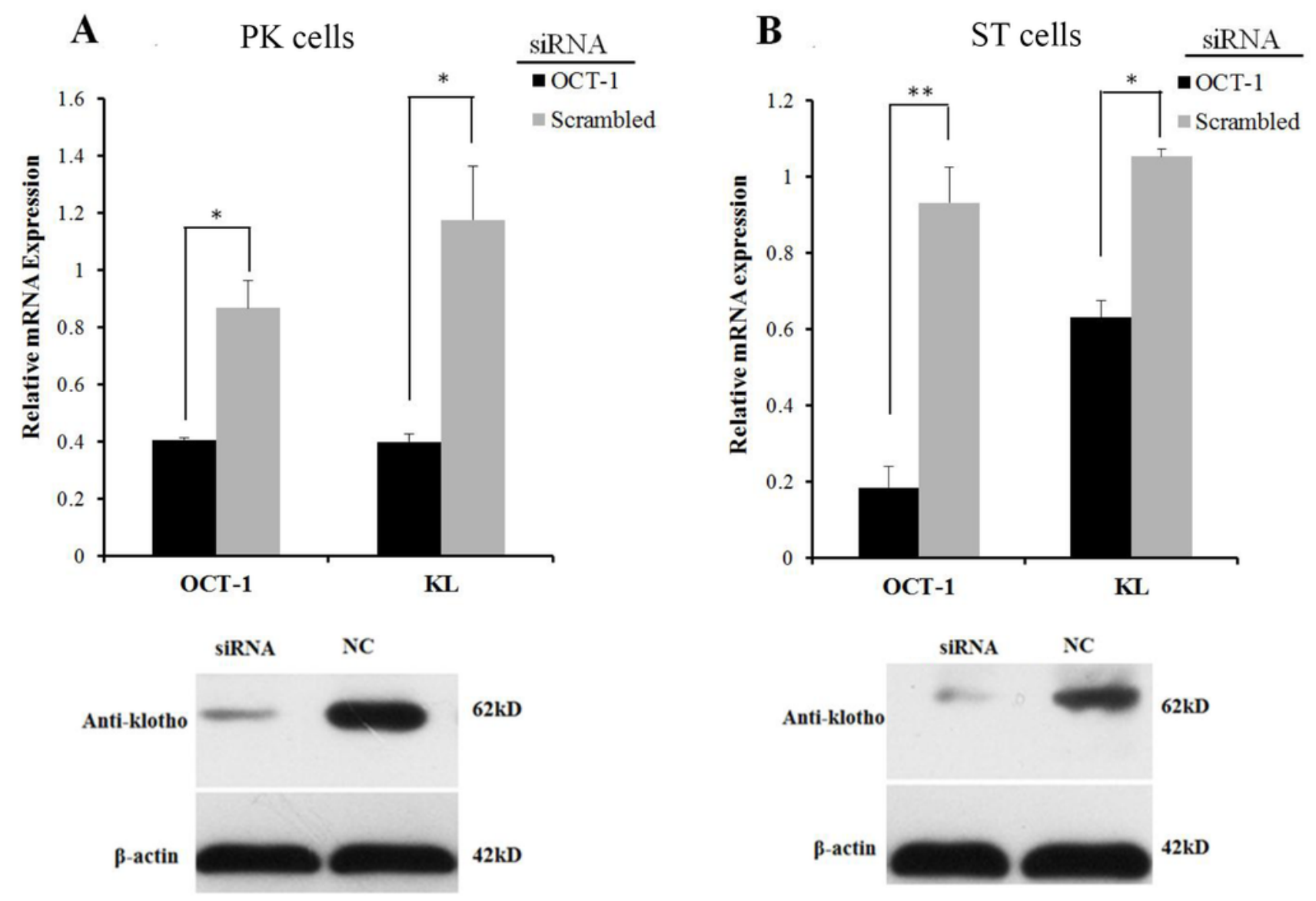
4

Binding of OCT-1 with $K L$ intron 1 was analyzed by EMSA and ChIP.

(A) The probe was incubated with nuclear extract in the absence or presence of 100-fold excess of various competitor probes (mutant or non-labeled probe) or anti-OCT-1. The specific super-shift (DNA-protein-antibody complex) bands were both observed in PK and ST cells. The sequences of various probes were demonstrated under the panel. (B) ChIP assay of OCT-1 binding to the $K L$ intron 1 in PK cells and ST cells. The interaction of OCT-1 in vivo with $K L$ intron region was determined by chromatin immunoprecipitation analysis. DNA isolated from immune-precipitated material was amplified by PCR to amplify KL fragement. Total chromatin was used as the input. Normal mouse IgG was used as a negative control.

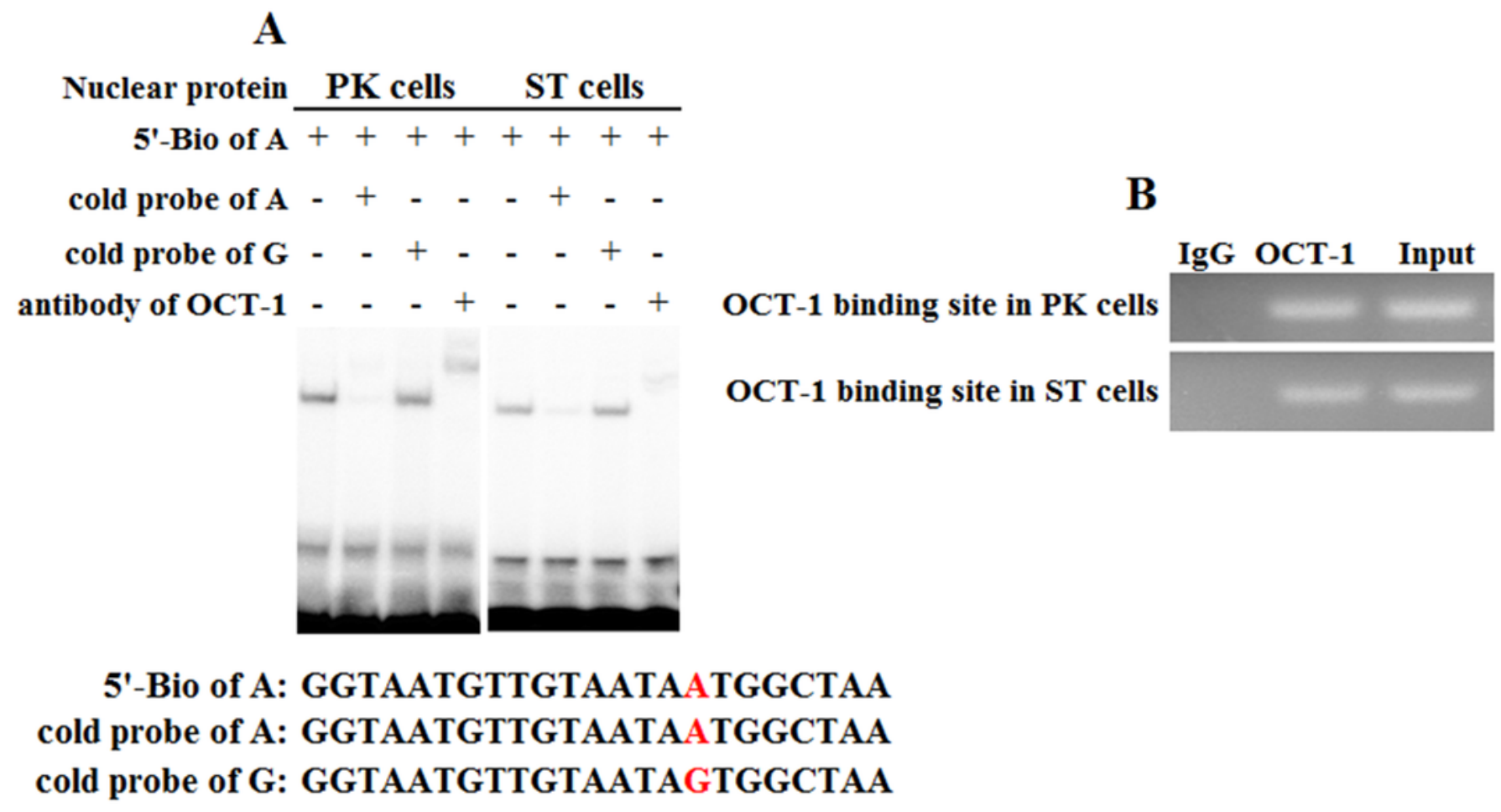




\section{Table 1 (on next page)}

Primers and DNA oligos used in this study. 
1 Table 1. Primers and DNA oligos used in this study.

\begin{tabular}{|c|c|c|c|}
\hline Primer & Primer sequence $\left(5^{\prime}-3^{\prime}\right)$ & $\begin{array}{c}\text { Amplicon } \\
\text { Length (bp) }\end{array}$ & $\begin{array}{l}\text { Tm } \\
\left({ }^{\circ} \mathrm{C}\right)\end{array}$ \\
\hline 5'-Bio of $\mathrm{A}(+)$ & GGTAATGTTGTAATAATGGCTAA & & \multirow{2}{*}{60} \\
\hline 5'-Bio of A (-) & TTAGCCATTATTACAACATTACC & & \\
\hline cold probe of $\mathrm{A}(+)$ & GGTAATGTTGTAATAATGGCTAA & & \multirow{2}{*}{60} \\
\hline cold probe of A (-) & TTAGCCATTATTACAACATTACC & & \\
\hline cold probe of $\mathrm{G}(+)$ & GGTAATGTTGTAATAGTGGCTAA & & \multirow{2}{*}{60} \\
\hline cold probe of G (-) & TTAGCCACTATTACAACATTACC & & \\
\hline$K L \_C h I P \_P F$ & TGAAGACCACTGCTACACACTT & & \multirow{2}{*}{59} \\
\hline$K L \_C h I P \_P R$ & AGCAAACAGGTTTTGTGGAGC & & \\
\hline & CGGGGTACCTTGTTGGATGTTTTGTT & \multirow{4}{*}{193} & \multirow{4}{*}{58} \\
\hline$K L \_\mathrm{D} 1 \_\mathrm{PF}$ & TGTCTAGCTAGC & & \\
\hline$K L \_\mathrm{D} \_\mathrm{PR}$ & CGACGCGTCCCTGTGAAGGCTTGTTT & & \\
\hline & CGGGGTACCTATGAGGAGGTGGGTT & & \\
\hline$K L \_\mathrm{D} 2 \_\mathrm{PF}$ & GGCTAGCTAGC & \multirow[t]{3}{*}{433} & \multirow[t]{3}{*}{59} \\
\hline$K L \_\mathrm{D} \_\mathrm{PR}$ & CGACGCGTCCCTGTGAAGGCTTGTTT & & \\
\hline & CGGGGTACCCACTTAАCCТCTTATTC & & \\
\hline$K L \_\mathrm{D} 3 \_\mathrm{PF}$ & TTGAGTTACTAGCTAGC & \multirow[t]{3}{*}{614} & \multirow[t]{3}{*}{59} \\
\hline$K L \_$D_PR & CGACGCGTCCCTGTGAAGGCTTGTTT & & \\
\hline & CGGGGTACCACATAAAAGTTAGAAA & & \\
\hline$K L \_$D4_PF & ATCAGAGAACTAGCTAGC & \multirow[t]{2}{*}{850} & \multirow[t]{2}{*}{59} \\
\hline$K L \_$D_PR & CGACGCGTCCCTGTGAAGGCTTGTTT & & \\
\hline$O C T 1 \_q P C R \_P F$ & TGAACAATCCGTCAGAAACC & \multirow{2}{*}{196} & \multirow{2}{*}{58} \\
\hline$O C T 1 \_q P C R \_P R$ & TGAGCAGCAGCCTGTAAACT & & \\
\hline$K L \_q P C R \_P F$ & ACCCGTATTTATTGATGGAGAC & \multirow{2}{*}{173} & \multirow{2}{*}{57} \\
\hline$K L \_q P C R \_P R$ & GGAACTTCATCTGAGGGTCTAA & & \\
\hline$K L \_$intron1_ChIP_PF & GCCGTAGATAATTGAAGC & \multirow{2}{*}{130} & \multirow{2}{*}{50} \\
\hline$K L \_$intron1_ChIP_PR & TCTGTGGTAGCAAACAGG & & \\
\hline$K L \_$intron2_ChIP_PF & GCCAGTGTAAGGTGTTACC & \multirow{2}{*}{114} & \multirow{2}{*}{51} \\
\hline$K L \_$intron2_ChIP_PR & ATTCTCCAAAGAAGACATACA & & \\
\hline$K L \_$intron3_ChIP_PF & CAAGATTGTACCGTGGAG & \multirow{2}{*}{171} & \multirow{2}{*}{50} \\
\hline$K L$ intron3_ChIP $P R$ & GGTCATTTGACATCATTCT & & \\
\hline
\end{tabular}

2 Protective bases and induced enzyme sites were in italic and bold respectively. 